Check for updates

Cite this: RSC Adv., 2018, 8, 20295

Received 30th April 2018 Accepted 24th May 2018

DOI: $10.1039 / c 8 r a 03702 g$

rsc.li/rsc-advances

\section{Studies of the binding properties of the food preservative thiabendazole to DNA by computer simulations and NMR relaxation}

\author{
Qiaomei Sun, ${ }^{a}$ Zili Suo, ${ }^{a}$ Hongyu Pu, ${ }^{a}$ Peixiao Tang, (iD *a Na Gan, ${ }^{a}$ Ruixue Gan, ${ }^{a}$ \\ Yuanming Zhai, ${ }^{\text {bb }}$ Xiaohui Ding ${ }^{a}$ and Hui Li (iD a
}

\begin{abstract}
Thiabendazole (TBZ) is a commonly used food preservative and has a wide range of anthelmintic properties. In this study, computer simulations and experiments were conducted to investigate the interaction mechanism of TBZ and herring sperm DNA (hsDNA) at the molecular level. Molecular docking showed that TBZ interacted with DNA in groove mode and bound in A-T and C-G base pair regions. Molecular dynamics (MD) was used to evaluate the stability of the TBZ-DNA complex and found that the three phases in $M D$ and the hydrogen bonds helped maintain the combination. NMR relaxation indicated that TBZ had a certain affinity to hsDNA with a binding constant of $462.43 \mathrm{~L} \mathrm{~mol}^{-1}$, and the thiazole ring was the main group bound with DNA. Results obtained from fluorescence experiments showed that the binding of TBZ and hsDNA was predominantly driven by enthalpy through a static quenching mechanism. Circular dichroism and viscosity measurements proved the groove binding mode. The FTIR results clarified the conformational changes of DNA, that the DNA helix became shorter and compact, and the DNA structure transformed from B-form to A-form.
\end{abstract}

\section{Introduction}

Thiabendazole [2-(4-thiazolyl) benzimidazole, TBZ] is a commonly used food preservative that is widely applied in various fruits and vegetables to slow down putridity caused by fungi. ${ }^{1}$ The structure of TBZ was shown in Fig. 1. Although the use of TBZ can provide benefits, its unreasonable use or excessive consumption leads to health risks; for example, TBZ can disrupt microtubules and induce anaphase-telophsae chromosomal aberrations., ${ }^{2,3}$ Thus, many researchers have focused on the binding properties of food additives and

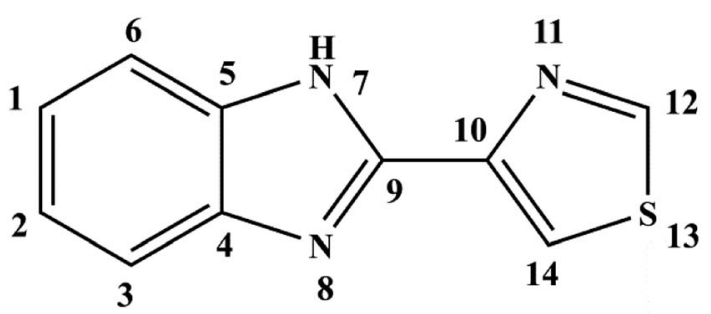

Fig. 1 Structure of thiabendazole (TBZ).

\footnotetext{
${ }^{a}$ School of Chemical Engineering, Sichuan University, Chengdu 610065, China. E-mail: tangpeixiao@126.com; Fax: +86028 85401207; Tel: +8602885405220

${ }^{b}$ Analytical \& Testing Center, Sichuan University, Chengdu 610065, China. E-mail: yuanmingzhai@scu.edu.cn
}

physiological macromolecules to preliminarily evaluate the safety and physiological properties of food additives. ${ }^{4-6}$

Deoxyribonucleic acid (DNA) is an important biomacromolecule that has potential therapeutic activity against many diseases, given that it is the pharmacological target of many drugs. ${ }^{7-9}$ The bindings of ligand and DNA have been indicated to affect DNA duplication and culminating transcription. ${ }^{10}$ The non-covalent interaction binding mode between ligands and the double helix of DNA include (i) groove binding where the ligand is located outside of the DNA helix groove with hydrogen bonds and (or) van der Waals interactions, and (ii) intercalation binding between ligand and the stacked base pairs of DNA, which usually have a greater impact on the structure and function of DNA compared with groove binding. ${ }^{11,12}$ The DNA damage and genotoxic and mutagenic effects of some food additives have been recently considered, and some of their adverse effects on DNA have been revealed. ${ }^{13}$ Moreover, there are some studies indicated that the difference in DNA structure had some effect on the binding process of ligand and receptor. ${ }^{\mathbf{1 4 1}}$ Hence, studies on the interaction between food additives and DNA can provide theoretical knowledge for the physiological properties of food additives and are beneficial to promote the development of safe food preservatives.

Receptor-ligand interactions have caught many researchers' attention, especially in the field of chemistry and life sciences. Many methods have developed to investigate these interactions. ${ }^{16-18}$ Among these methods, computer simulations 
including molecule docking and molecular dynamic (MD) simulation, are a powerful tool to determine the orientation and binding site for visualization of the binding properties and to monitor the dynamics behavior of the ligand/receptor structures during combination. ${ }^{\mathbf{1 9 , 2 0}}$ Complementarily, nuclear magnetic resonance (NMR) provides reasonable and reliable data to clarify the binding properties of ligand at the molecular level by identifying ligand epitope and structure affinity. ${ }^{21,22}$ Relaxation time technique is an effective method to investigate the binding affinity and dynamic properties on the basis of the quantitative analysis of spin-lattice relaxation rate changes of proton. ${ }^{23-25}$

Given that the binding mode of TBZ and calf thymus DNA (ctDNA) had been researched by fluorescence, UV absorbance and circular dichroism (CD) spectra, ${ }^{26}$ the main aim of this work was to further investigate the binding mechanism between TBZ and herring sperm DNA (hsDNA) and probe into the effects on the binding properties for different DNA models by combining theories and experiments. The interaction characteristics of TBZ-hsDNA would be clarified from the point of the superior groups and proton affinity abilities of TBZ. Molecular docking and MD were conducted to estimate the binding mode, binding region, and structural characteristics and to evaluate the dynamics behavior and stability of TBZ-DNA complex. NMR relaxation experiments probed into the binding mechanism (including binding constant and affinity) from the point of ligand molecule. The binding mode and structural feature of TBZ to hsDNA were corroborated by spectroscopy. This study will help us understand the physiological pharmacokinetic behavior of TBZ at the molecular level and will provide interesting insights into the interactions of food additives and DNA.

\section{Materials and methods}

\subsection{Reagents and chemicals}

Herring sperm DNA (hs-DNA) was obtained from Solarbio (Beijing, China) and was dissolved in a phosphate buffer solution (PBS, $0.01 \mathrm{M}$ ). The purity of the DNA was checked by a ratio of UV absorbance at 260 and $280 \mathrm{~nm}$. The ratio was larger than 1.8 , indicating that the hs-DNA was sufficiently free from any protein contamination. ${ }^{27}$ The stock solution of the DNA was stored in the dark at $4{ }^{\circ} \mathrm{C}$ and used within a week.

TBZ was purchased from Aladdin Chemical Reagent (Shanghai, China). All of the other reagents were of analytical grade and used without further purification. Ultrapure water was used throughout the experiment.

\subsection{Molecule docking and molecule dynamics simulation}

FlexX docking program interfaced within LeadIT was used to examine possible binding modes and active site of TBZ and DNA. $^{20}$ The 3D structure of TBZ was obtained from PubChem (Pub-Chem CID: 5430), and the crystallographic structure of DNA duplex (PBD ID: 1BNA) was downloaded from PCSB protein data bank with the sequence of (CGCGAATTCGCG)2. The hydrogen atoms of DNA were added using Receptor Wizard module. The FlexX hybrid algorithm was selected to perform the docking with a radius of $20 \AA$ binding site definition. The docking binding modes of the top five highest scored docking poses were evaluated, and the optimal binding poses were used for molecular dynamics simulation.

MD simulations were conducted using YASARA v16.7.22 package with the AMBER14 force field, to determine the optimal binding conformation and assess the system stability. ${ }^{19}$ The simulation was performed at a NPT ensemble with a constant temperature at $298 \mathrm{~K}$ and constant pressure (1 bar) at $\mathrm{pH} 7.4$. Counter ions $\left(\mathrm{Na}^{+}\right.$or $\left.\mathrm{Cl}^{-}\right)$were added by randomly replacing water molecules to obtain a charge-neutral system. Periodic boundary conditions were applied in the MD process. Simulations were carried out after initial energy minimization procedures were conducted, by using a pre-defined macro (md_run) within the YASARA package. According to the MD trajectories, root mean square deviations (RMSD) were analyzed.

\subsection{NMR measurements}

All data were acquired using a Bruker Advance $400 \mathrm{MHz}$ NMR spectrometer, operated at $400.13 \mathrm{MHz}$ at $25{ }^{\circ} \mathrm{C}$. The spin-lattice relaxation rates were measured using the standard inversionrecovery $\left(180^{\circ}-\tau-90^{\circ}-t\right)_{n}$ sequence. The $\tau$ values used for the experiments were $0.01,0.02,0.04,0.06,0.1,0.2,0.4,0.8,1,3,5$, 7,20 , and $25 \mathrm{~s}$. The delay time $t$ in this case was $25 \mathrm{~s}$. The $180^{\circ}$ selective inversion of the proton spin population was obtained through a selective soft Gaussian perturbation pulse (width: 20 ms, power: $60 \mathrm{~dB}$ ) with an excitation width of about $45 \mathrm{~Hz}$. The addition of HSA did not change the viscosity of the system, and the observed $R_{i}^{\text {se }}$ enhancements were mainly affected by the ligand-protein complex formation. All NMR data processing and analyses were performed using Topspin 2.1 software (Bruker BioSpin, Ltd.).

\subsection{Fluorescence spectroscopy}

Fluorescence intensities were recorded using a Cary Eclipse fluorophotometer (Varian, USA) equipped with $0.1 \mathrm{~cm}$ quartz cells. Each solution was left to stand for 1 hour to achieve equilibrium. The wavelength range from $310 \mathrm{~nm}$ to $550 \mathrm{~nm}$ with excitation wavelength at $300 \mathrm{~nm}$ was selected. The slit widths for both excitation and emission were set to 10 .

\subsection{Fluorescence lifetime measurements}

The fluorescence lifetimes were determined by time-correlated single photon counting (TCSPC) using a Horiba Jobin Yvon FluoroMax-4 spectrofluorometer (HORIBA, FRA). The timeresolved intensity decay of TBZ was measured in the absence and presence of hs-DNA in PBS buffer $(\mathrm{pH}=7.4)$ at an excitation and emission wavelengths of $317 \mathrm{~nm}$ and $360 \mathrm{~nm}$. The TBZ concentration was fixed at $2.0 \times 10^{-5} \mathrm{~mol} \mathrm{~L}^{-1}$, and the hs-DNA concentrations were varied from $4.0 \times 10^{-4} \mathrm{~mol} \mathrm{~L}^{-1}$ to $1.2 \times$ $10^{-3} \mathrm{~mol} \mathrm{~L}^{-1}$ at room temperature.

\subsection{Circular dichroism spectra}

Far UV-CD spectra were recorded using a Hitachi-F7000 fluorescence spectrometer with a $1.0 \mathrm{~cm}$ path length quartz cell at 
$298 \mathrm{~K}$. The spectra were obtained at wavelength ranging from $190 \mathrm{~nm}$ to $320 \mathrm{~nm}$ with a step size of $1.0 \mathrm{~nm}$, and average time of 0.5 s. The concentration of hsDNA was fixed at $4.0 \times$ $10^{-4} \mathrm{~mol} \mathrm{~L}^{-1}$, whereas that of TBZ ranged from $0 \mathrm{M}$ to $4.0 \times$ $10^{-5} \mathrm{~mol} \mathrm{~L}^{-1}$. Each CD spectrum was recorded from the average values of three scans.

\subsection{Viscosity measurements}

Viscosity was measured using the Discovery HR-2 hybrid rheometer (TA Instruments, New Castle, DE, USA) at $298 \mathrm{~K}$. The concentration of hsDNA was fixed at $4.0 \times 10^{-5} \mathrm{~mol} \mathrm{~L}^{-1}$ and added with different concentrations of TBZ. The viscosities of samples were calculated by averaging three measurements. The values of relative specific viscosity $\left(\eta / \eta_{0}\right)^{1 / 3}$ were plotted against the ratios of $[\mathrm{TBZ}] /[\mathrm{hsDNA}](r=0.0,0.1,0.2,0.4,0.8,1.2)$, where $\eta_{0}$ and $\eta$ are the viscosity of DNA in the absence and presence of TBZ, respectively.

\subsection{FTIR spectrum measurements}

The FTIR spectrum measurements were conducted on a Nicolet6700 FTIR spectrometer (Thermo, USA) with a smart OMNIsampler accessory. The FTIR spectra of TBZ with hs-DNA at molar ratios of TBZ to hs-DNA of $1: 0,1: 20,1: 40$, and $1: 80$ were obtained. All the spectra were collected in the range of
1800-700 $\mathrm{cm}^{-1}$ by averaging 128 scans with a resolution of $4 \mathrm{~cm}^{-1}$. All the measurements were conducted at room temperature, and the background spectra were collected before each measurement.

\section{Results and discussion}

\subsection{Computer simulation analysis of the binding of TBZ and DNA}

3.1.1 Molecular docking model research. The possible binding mode, binding region and structural characteristics of TBZ and DNA were predicted by molecular docking. The docking clusters in Fig. 2 were clearly divided into two symmetrical groups that were constituted by A-T and $\mathrm{C}-\mathrm{G}$ base pairs. All docking results showed that the binding mode of TBZ and DNA was groove interaction. The top five highest-scored docking poses were selected to further probe into the optimal binding pose and structural characteristics of the binding (Fig. 2). The five docking poses all presented that TBZ was surrounded by both A-T and C-G base pairs, instead of being located in pure A$\mathrm{T}$ or C-G region. Moreover, hydrophobic forces and hydrogen bonds were the main forces for the binding of TBZ and DNA, which indicated a groove interaction rather than an intercalation. The nitrogen atoms on the thiazole and imidazole ring of TBZ formed hydrogen bonds with the amino and (or) nitrogen

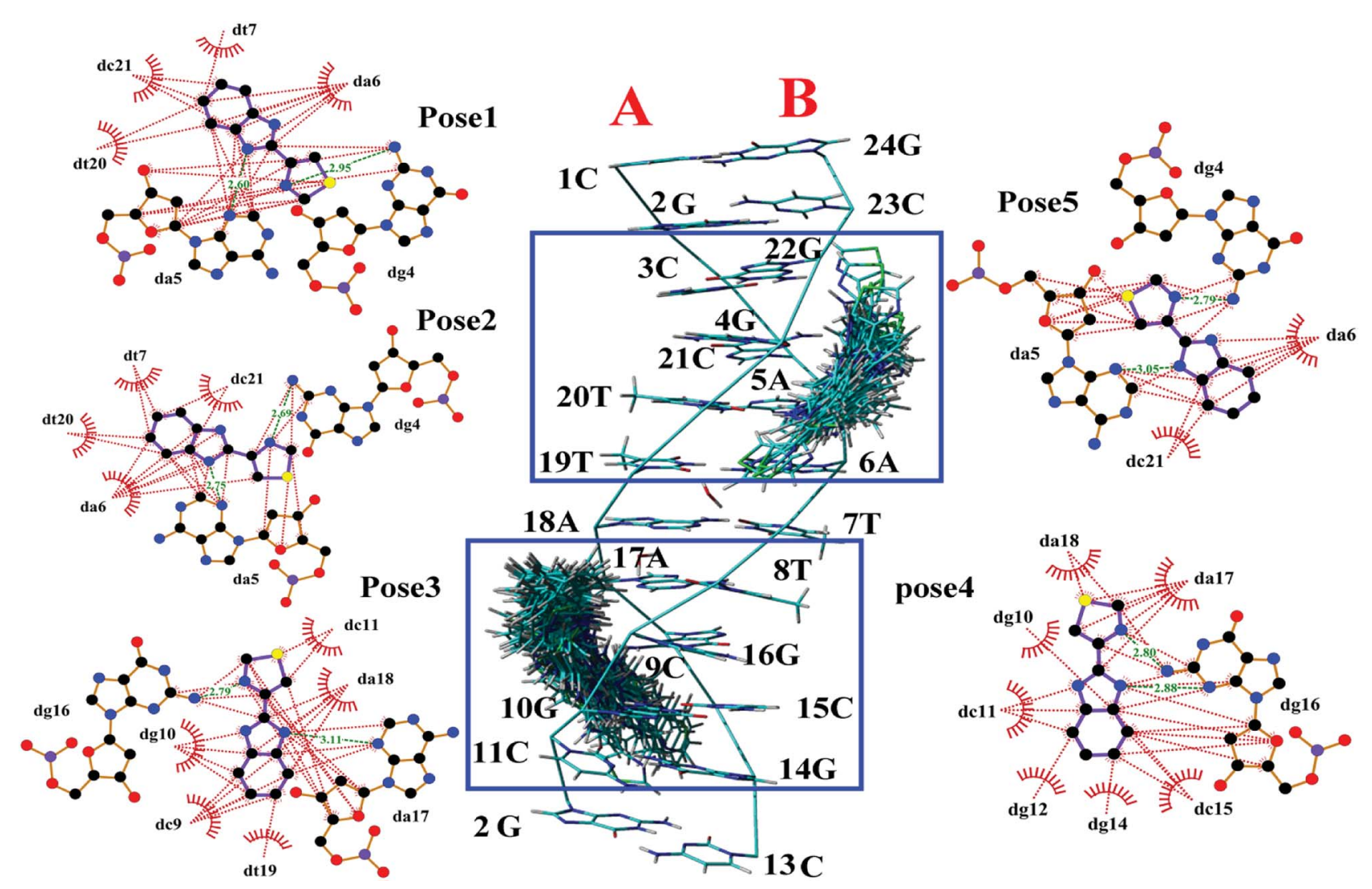

Fig. 2 Middle image was the cluster analysis for conformations of the TBZ docking with numbered DNA [PBD ID: 1BNA, (CGCGAATTCGCG)2] generated by FlexX. The two chains ( $\mathrm{A}$ and $\mathrm{B}$ ) and base sequence of DNA were labeled. The five surrounded images were the top five highestscored docking poses generated by LigPlot, wherein the hydrophobic interaction was red dashed, and the hydrogen bonds were green dashed with distance. 


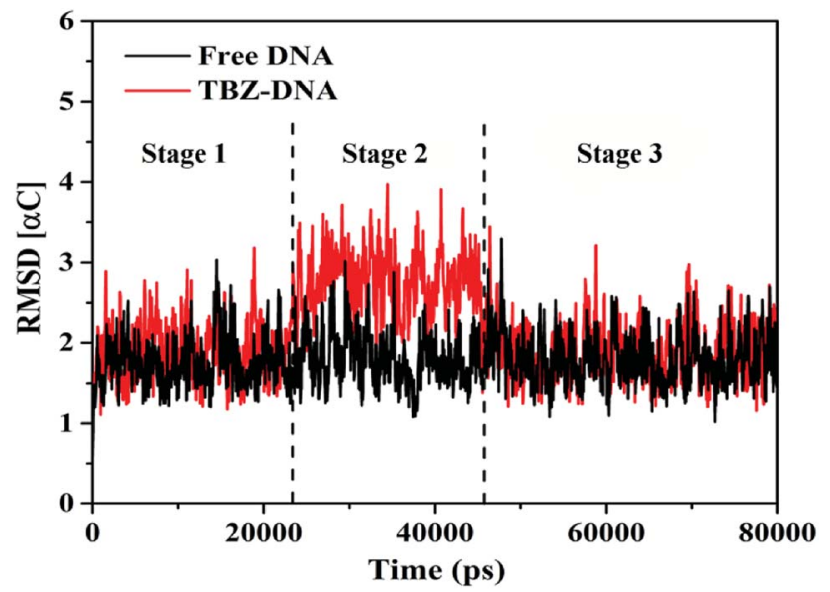

Fig. 3 Root mean square deviations of the complex systems in the MD simulations.

atoms on the pyrimidine ring of adenine and guanine. This phenomenon indicated that adenine and guanine were the main base pairs that participated in the interaction. Given the similar binding characteristics of the top five binding poses, the top one was selected for molecular dynamical simulation to evaluate the stability of the interaction and explore the steady binding conformation.

\subsubsection{Analysis of molecular dynamics}

3.1.2.1. Analysis of the systematic stabilities using RMSD. In this study, MD simulations were performed under simulated physiological conditions to further understand the stability and dynamics behavior of TBZ-DNA complex in different timescales. Root mean square deviations, which is deviation statistics on behalf of the structural changes and the atomic fluctuation from the initial position, is a parameter for assessing the stability of simulation system..$^{28}$ The RMSD values of $\alpha$ carbon atoms for the free DNA and TBZ-DNA complex systems versus simulation time were showed in Fig. 3. The RMSD values for free DNA fluctuated in a balanced position throughout the simulation and thus indicated the stable double helix structure of DNA. Compared with that in blank control system, the RMSD in TBZ-DNA systems displayed an apparent disturbance and eventually reached plateau at approximately $46 \mathrm{~ns}$. Thus, TBZ could steadily bind with DNA and stayed at a stable binding position after a slight structural drift and low atomic fluctuations.

3.1.2.2. Dynamics behavior analysis of trajectory. According to the fluctuation of RMSD in $80 \mathrm{~ns}$, the molecular dynamics process can be divided into three stages for trajectory analysis to evaluate the dynamics behavior of the binding (Fig. 3). The first stage (Stage 1) was from the initial state to $24 \mathrm{~ns}$, the second stage (Stage 2) was from $24 \mathrm{~ns}$ to $46 \mathrm{~ns}$, and the third stage (Stage 3) was from $46 \mathrm{~ns}$ to $80 \mathrm{~ns}$. A representative trajectory for each stage was selected, specifically, $0 \mathrm{~ns}, 35 \mathrm{~ns}$, and $80 \mathrm{~ns}$ represented Stage 1, Stage 2, and Stage 3, respectively. As shown in Fig. 4, the TBZ molecule moved from the center of the DNA to the edge area in the MD simulation. In the initial conformation (Stage 1, time $=0 \mathrm{~ns}$ ), the TBZ molecule bound in the border area of A-T and C-G base pairs of DNA, and formed two hydrogen bonds with adenine and guanine in the distance of 2.99 and $2.95 \AA$, respectively. In Stage 2 (time $=35 \mathrm{~ns}$ ), the hydrogen bond between TBZ and DNA fractured, and the TBZ molecule drifted from the initial binding position and was maintained by hydrophobic interaction with DNA. In the final stable state (Stage 3, time $=80 \mathrm{~ns}$ ), TBZ steadily bound in the terminal region enriched with $\mathrm{A}-\mathrm{T}$ base pairs by forming hydrogen bonds with cytosine and guanine in the distances of 2.71 and $3.14 \AA$, respectively. In conclusion, TBZ bound to the groove position of DNA comprising A-T and C-G base pairs. Hydrogen bonds and hydrophobic interaction were the main forces for maintaining this combination.

The hydrogen bond numbers of TBZ-DNA system were analyzed throughout the MD simulation and were shown in Fig. 5. The numbers of hydrogen bond ranged from 0 to 2, and the variation of hydrogen bonds number could be divided into three stages similar to RMSD. In Stages 1 and 3, the numbers of hydrogen bond ranged from 1 to 2, whereas that in Stage 2 ranged from 0 to 1 . The results indicated that hydrogen bonds played an important role in the binding and stabilities of TBZ and DNA.

\subsection{Analysis of the binding mechanism of TBZ-DNA system}

3.2.1. Theory of NMR relaxation study. The proton spin relaxation rate of the small molecules was measured using NMR spectroscopy. The selective spin-lattice relaxation rates $\left(R^{\mathrm{se}}\right)$ are sensitive to the affinity of ligand with and without receptors. The binding process of the ligand-receptor can be described as follows:

$$
\mathrm{M}+\mathrm{L}=\mathrm{ML}
$$

When the ligand-receptor reach equilibration, the thermodynamic equilibrium constant can be written as $K=[\mathrm{ML}] /[\mathrm{M}]$ [L]. Under conditions of fast chemical exchange between free and bound environments, the $R^{\text {se }}$ can be expressed by the following equation: ${ }^{24}$

$$
\Delta R^{\mathrm{se}}=\frac{K R_{\mathrm{b}}^{\mathrm{se}}}{(1+K[\mathrm{~L}])}\left[\mathrm{M}_{0}\right]
$$

where $\Delta R^{\mathrm{se}}=R_{\mathrm{obs}}^{\mathrm{se}}-R_{\mathrm{f}}^{\mathrm{se}}, R_{\mathrm{obs}}^{\mathrm{se}}$ is the experimentally determined selective relaxation rate, $R_{\mathrm{f}}^{\mathrm{se}}$ and $R_{\mathrm{b}}^{\mathrm{se}}$ are the selective spin-lattice relaxation rates in free and bound states, respectively. The affinity index needs be normalized to the free ligand relaxation rate due to the differences in the portions dynamics of the molecule caused by the different correlation times and modulation of the dipolar interactions between protons at different positions and spin densities on the ligand proton. The normalization of $\Delta R^{\mathrm{se}}=R_{\mathrm{obs}}^{\mathrm{se}}-R_{\mathrm{f}}^{\mathrm{se}}$ to $R_{\mathrm{f}}^{\text {se }}$ cancels the effects of the above-mentioned factors on the interaction between ligand and macromolecule and leads to a normalized affinity index:

$$
\Delta R_{\mathrm{N}}^{\mathrm{se}}=\frac{K R_{\mathrm{b}}^{\mathrm{se}}}{(1+K[\mathrm{~L}]) R_{\mathrm{f}}^{\mathrm{se}}}\left[\mathrm{M}_{0}\right]
$$




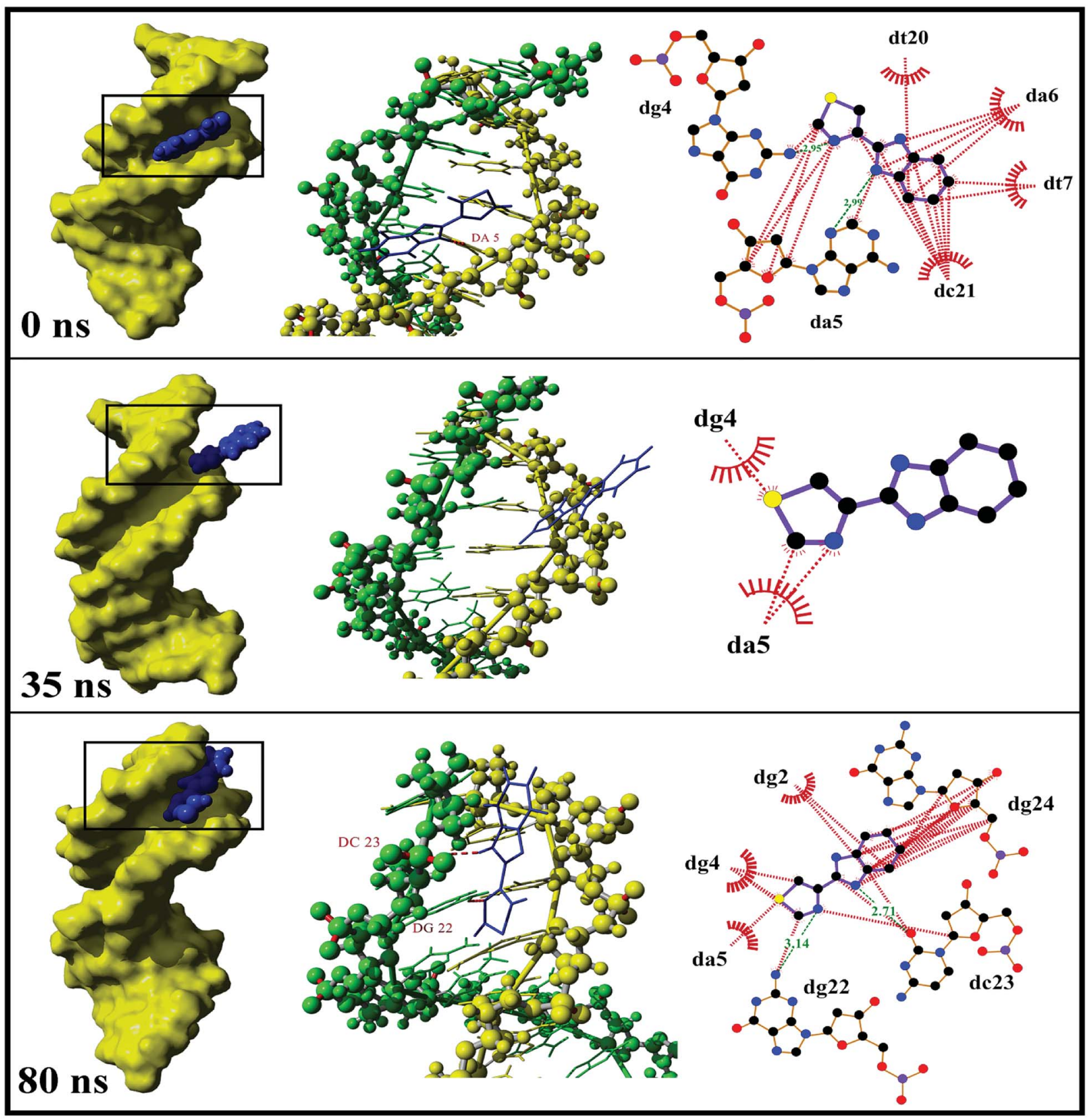

Fig. 4 Trajectory analysis of three stages at 0,35 , and $80 \mathrm{~ns}$. Left images showed the binding region and binding mode of TBZ to DNA, where DNA was showed as molecular surface and TBZ was spherical model. Middle images were the interaction conformations of complex, in which the hydrogen bonds were red dotted line and the base formed hydrogen bond with TBZ was labeled. Right images showed the schematic representation of Ligplot software, wherein hydrophobic interaction was colored red and hydrogen bond colored green with distance.

The normalized relaxation rate $\Delta R_{\mathrm{N}}^{\mathrm{se}}$ versus the macromolecule concentration $\left[\mathrm{M}_{0}\right]$ is characterized by a positive linear correlation. The slope of the straight line passing through the origin of the axes is $\left[A^{\mathrm{N}}\right]_{\mathrm{L}}^{\mathrm{T}}$ :

$$
\left[A^{\mathrm{N}}\right]_{\mathrm{L}}^{\mathrm{T}}=\frac{K R_{\mathrm{b}}^{\mathrm{se}}}{(1+K[\mathrm{~L}]) R_{\mathrm{f}}^{\mathrm{se}}}
$$

which is defined as the "normalized affinity index" $\left(\mathrm{L} \mathrm{mol}^{-1}\right)$. This value remains constant at a specified temperature and ligand concentration. Moreover, the normalized relaxation rate $\Delta R_{\mathrm{N}}^{\mathrm{se}}$ eqn (3) can be shown as:

$$
\frac{1}{\Delta R_{\mathrm{N}}^{\mathrm{sec}}}=\frac{R_{\mathrm{f}}^{\text {se }}}{K R_{\mathrm{b}}^{\text {se }}\left[\mathrm{M}_{0}\right]}+\frac{[\mathrm{L}] R_{\mathrm{f}}^{\text {se }}}{R_{\mathrm{b}}^{\text {se }}\left[\mathrm{M}_{0}\right]}
$$

Plotting $1 / \Delta R_{\mathrm{N}}^{\mathrm{se}}$ to the ligand concentration $[\mathrm{L}]$, the binding constant $K$ can be calculated. 


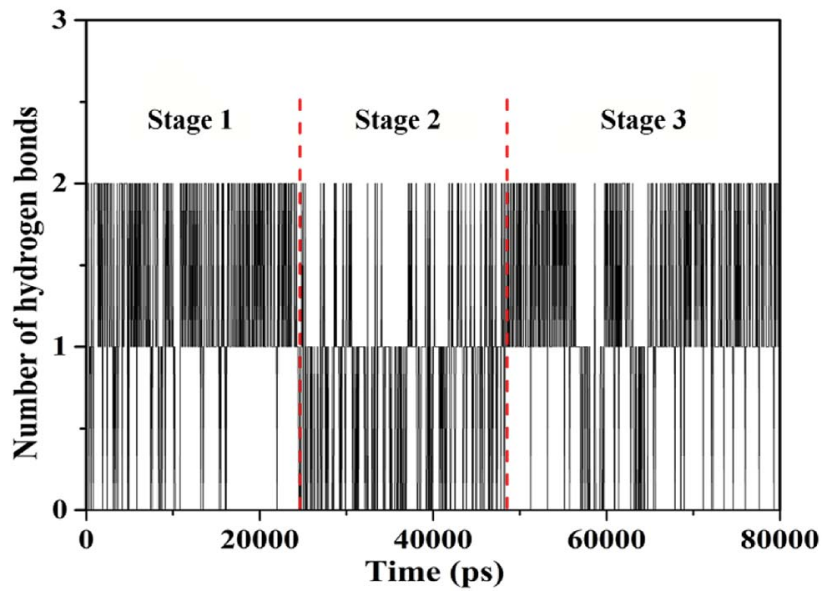

Fig. 5 The number of hydrogen bonds between TBZ and DNA throughout the MD simulations.

3.2.2. Analysis of the affinities and binding constant. The ${ }^{1} \mathrm{H}$ NMR spectrum of TBZ was shown in Fig. 6A. According to the results of computer simulations, three groups of hydrogen protons from the benzene ring and the thiazole ring $(\mathrm{H} 1 / \mathrm{H} 2$, $\mathrm{H} 14$, and H12) were selected to compare the binding abilities. The normalized affinity indexes $\left[A_{i}^{\mathrm{N}}\right]_{\mathrm{L}}^{\mathrm{T}}$ were fitted in Fig. $6 \mathrm{~B}$ to remove the effects of different correlation times and proton densities. The curves showed that the normalized selective relaxation rate of TBZ increased with the addition of DNA. For the $\mathrm{H} 1 / \mathrm{H} 2, \mathrm{H} 14$, and $\mathrm{H} 12$, the values of $\left[A_{i}^{\mathrm{N}}\right]_{\mathrm{L}}^{\mathrm{T}}$ for the TBZ-DNA system were $1396.23 \pm 254.19,33573.03 \pm 4120.80$, and $8498.93 \pm 5.52 \mathrm{~L} \mathrm{~mol}^{-1}$, respectively. The NMR results confirmed that the thiazole ring was the main group bound with DNA, and the affinity of benzene rings was weak due to the absence of hydrogen protons on the imidazole ring.

In order to ascertain the binding mechanism between TBZ and DNA, the binding constant was calculated according to eqn (5) in Fig. 6C. The binding constant of the TBZ-DNA complex was $462.43 \mathrm{~L} \mathrm{~mol}^{-1}$ at $298 \mathrm{~K}$, which was lower than that of the reported intercalate binding mode ranging from $10^{5} \mathrm{~L} \mathrm{~mol}^{-1}$ to $10^{6} \mathrm{~L} \mathrm{~mol}^{-1} .^{29}$ The low binding affinity of the TBZ and DNA served as an evidence for the non-intercalative binding mode.

3.2.3. Investigation of fluorescence emission spectrum. The emission spectra of TBZ at different concentrations of DNA were shown in the inner picture of Fig. 7. The maximum fluorescence intensity of TBZ decreased with the increase of DNA, indicating that the fluorophore of TBZ was affected with the added DNA. The quenching constant $\left(K_{\mathrm{sv}}\right)$ and binding constant $(K)$ were calculated by the linear Stern-Volmer equation ${ }^{\mathbf{3 0 , 3 1}}$ at four different temperatures $(298,303,310$, and $317 \mathrm{~K}$ ) and were presented in Table 1 . It is well-known that the decrease of $K_{\mathrm{sv}}$ with the increased temperatures implies that the fluorescence quenching mechanism is static quenching, whereas a reverse trend is dynamic quenching. ${ }^{32,33}$ Thus, the interaction between TBZ and hsDNA was static quenching.
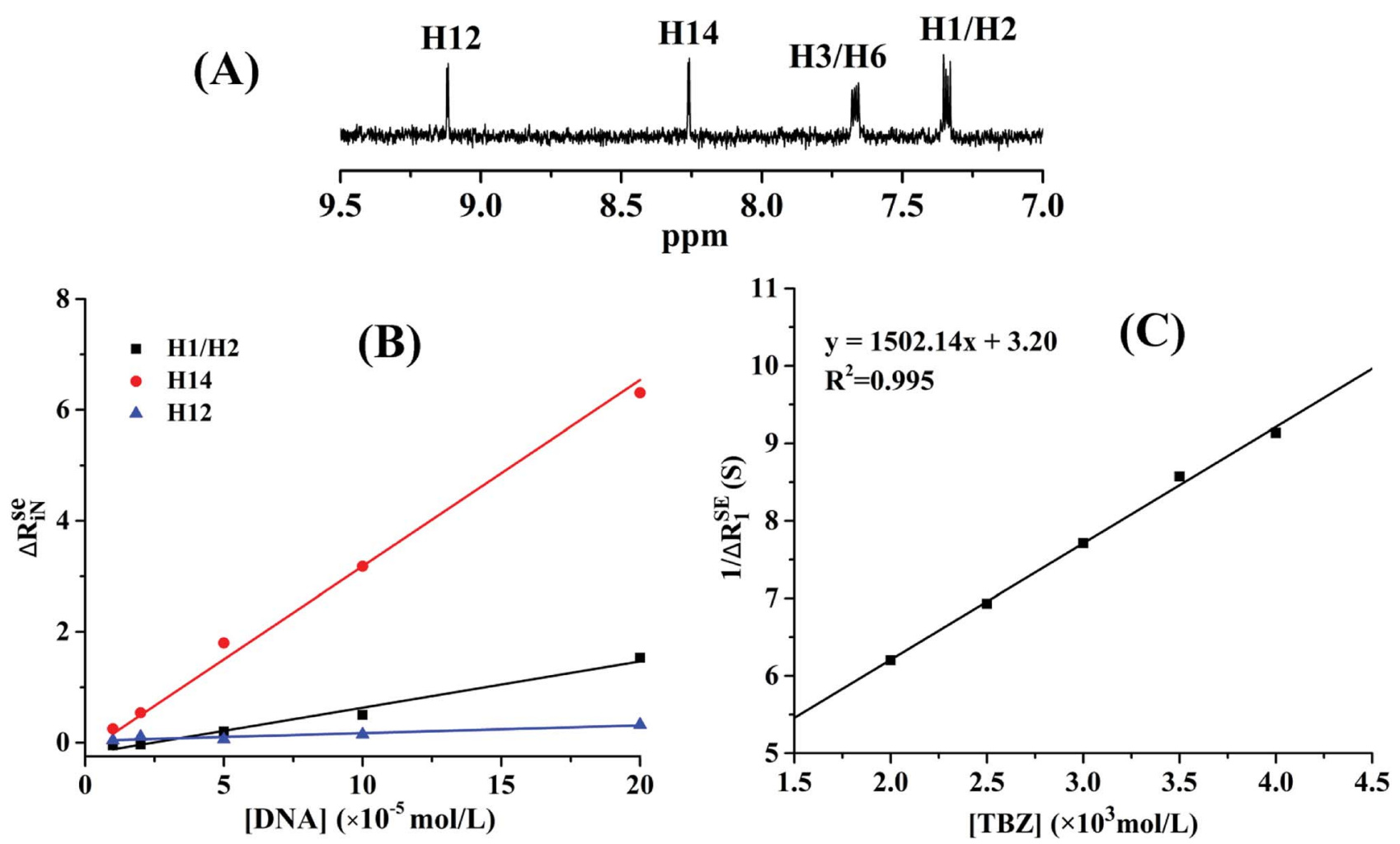

Fig. 6 (A) Proton NMR spectrum of thiabendazole in DMSO- $d_{6}$ solution. (B) Plots of normalized selective relaxation rate enhancements of H1/ $\mathrm{H} 2, \mathrm{H} 14$, and $\mathrm{H} 12$. [TBZ] $=2 \times 10^{-3} \mathrm{~mol} \mathrm{~L}^{-1}$, [DNA] $=1,2,5,10,20 \times 10^{-5} \mathrm{~mol} \mathrm{~L}^{-1}, \mathrm{pH}=7.4, T=298 \mathrm{~K}$. (C) Equilibrium constant of the TBZ-DNA system. $[\mathrm{DNA}]=2 \times 10^{-5} \mathrm{~mol} \mathrm{~L}^{-1},[\mathrm{TBZ}]=2,2.5,3,3.5,4 \times 10^{-3} \mathrm{~mol} \mathrm{~L}-1, \mathrm{pH}=7.4, T=298 \mathrm{~K}$. 


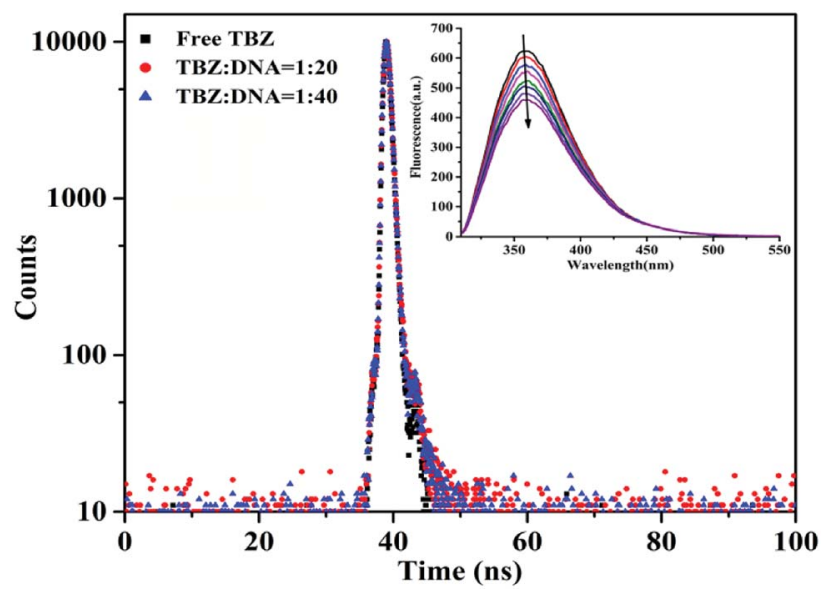

Fig. 7 The fluorescence decay curve of TBZ in the presence and absence of hs-DNA $\left(\lambda_{\mathrm{ex}}=310 \mathrm{~nm}\right.$ and $\left.\lambda_{\mathrm{em}}=360 \mathrm{~nm}\right)$. The inner figure is emission spectra of TBZ in different concentrations of DNA at $298 \mathrm{~K}$.

As shown in Table 1, the value of $K$ was $471.53 \mathrm{~L} \mathrm{~mol}^{-1}$ at 298 $\mathrm{K}$, which was similar with NMR results. The values of $K$ decreased with the increase of temperature, possibly indicating that the decomposition of the TBZ-DNA complex with the temperature increased. Moreover, the binding forces (including hydrogen bonds, electrostatic attraction, van der Waals forces, and hydrophobic interaction) were analyzed by the thermodynamic parameters using the Van't Hoff equation. ${ }^{\mathbf{1 4 , 3 4}}$ Hydrogen bonds and van der Waals forces played the main role in the interaction because of the negative $\Delta H$ and $\Delta S$. These values showed that the interaction was spontaneous and predominantly driven by enthalpy; these characteristics were consistent with the other non-intercalative binding. ${ }^{35}$ A previous study $^{\mathbf{2 6}}$ had shown that the binding was an entropy-driven process and maintained by hydrophobic force, the different thermodynamic behavior compared with this work might be due to the model selection of DNA from different sources.

3.2.4. Time-resolved fluorescence spectrum. Fluorescence lifetime measurement is an important method to distinguish between static and dynamic quenching of acceptor and ligand. In static quenching, the decay time of the free fluorophores does not change, whereas in dynamic quenching, the mean decay time of the entire population in the excited state decreases. ${ }^{36}$ In Fig. 7, the average lifetime of TBZ remained almost constant with the addition of hsDNA. This finding leaded to the conclusion of static quenching mechanism between TBZ and hsDNA, and was in good agreement with the fluorescence quenching analysis.

\subsection{Investigation of the binding mode}

3.3.1. Circular dichroism (CD) spectroscopy. $C D$ is a powerful tool to monitor the structure of DNA, which the characteristic peak of right-handed B form DNA was a positive peak at $\sim 276 \mathrm{~nm}$ (base stacking) and a negative peak at

Table 1 Quenching and thermodynamic parameters of TBZ-DNA systems at four different temperatures

\begin{tabular}{|c|c|c|c|c|c|c|c|c|c|}
\hline$T(\mathrm{~K})$ & $K_{\mathrm{sv}}\left(\mathrm{L} \mathrm{mol}^{-1}\right)$ & $R^{a}$ & $K_{\mathrm{a}}\left(\mathrm{L} \mathrm{mol}^{-1}\right)$ & $n$ & $R^{b}$ & $\Delta G\left(\mathrm{~kJ} \mathrm{~mol}^{-1}\right)$ & $\Delta H\left(\mathrm{~kJ} \mathrm{~mol}^{-1}\right)$ & $\Delta S\left[\mathrm{~J}\left(\mathrm{~mol}^{-1} \mathrm{~K}^{-1}\right)\right]$ & $R^{c}$ \\
\hline 298 & 275.63 & 0.991 & 471.56 & 1.094 & 0.996 & -15.220 & -30.779 & -52.385 & 0.975 \\
\hline 303 & 265.23 & 0.975 & 385.75 & 1.063 & 0.999 & -14.906 & & & \\
\hline 317 & 249.94 & 0.994 & 221.65 & 0.966 & 0.995 & -14.173 & & & \\
\hline
\end{tabular}

${ }^{a}$ The correlation coefficient for the $K_{\mathrm{sv}}$ values. ${ }^{b}$ Is the standard deviation for the $K$ values. ${ }^{c}$ The correlation coefficient for the van t Hoff plot.
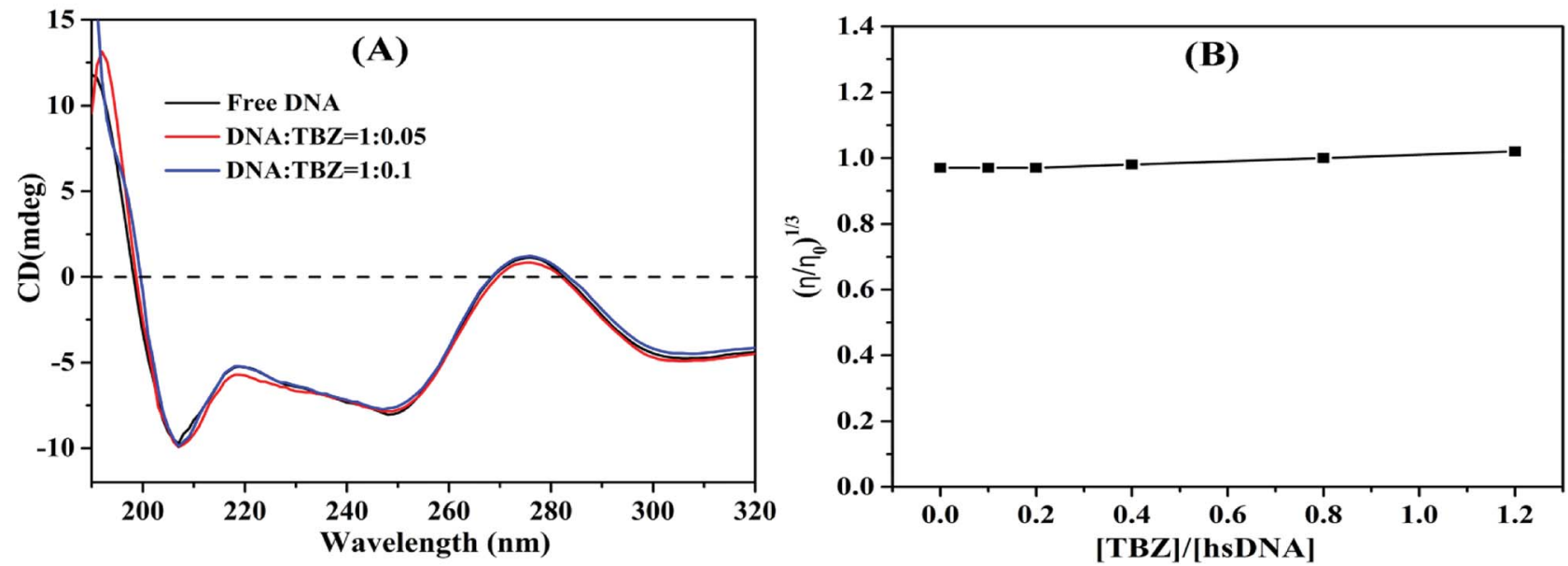

Fig. 8 (A) CD spectra of hsDNA with the increasing concentrations of TBZ. (B) Effect of increasing amounts of TBZ on the relative viscosity of hsDNA at $298 \mathrm{~K}$. 

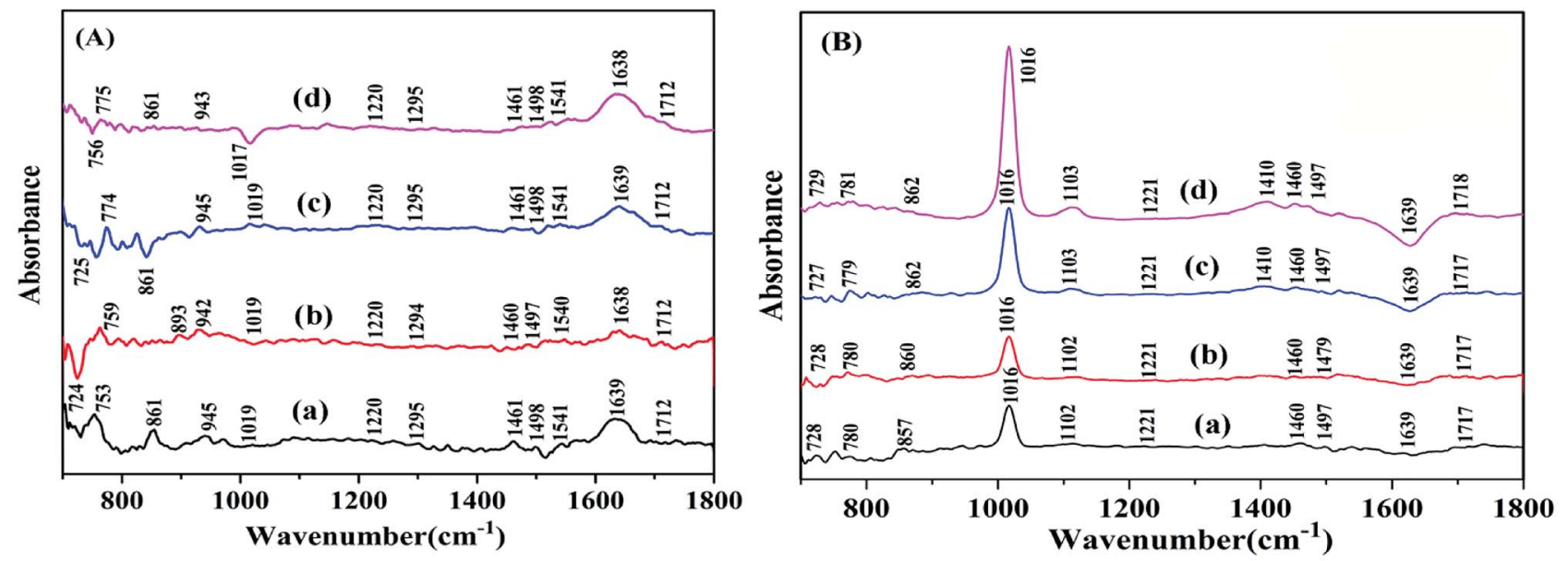

Fig. 9 (A) The difference spectra of free DNA (a) and [(DNA + TBZ) - TBZ]. (B) The difference spectra of free drug (a) and [(DNA + TBZ) - DNA]. The molar ratios ([TBZ] : [hsDNA]) for (b)-(d) were $1: 20,1: 40$ and $1: 80$, respectively.

$\sim 250 \mathrm{~nm}$ (right-handed helicity). ${ }^{37}$ The changes in the CD spectra are related to the binding mode; that is, intercalation affects both the positive and negative peaks, and groove interaction almost does not change the CD spectra of DNA. In this work, the CD spectra of hsDNA with and without TBZ were shown in Fig. 8A. The most probable binding mode of TBZ with hsDNA was groove binding because the addition of TBZ had minimal effect on the DNA structure.

3.3.2. Viscosity measurements. Viscosity measurement is a useful and effectively method to distinguish the binding mode of ligand-DNA interaction..$^{38}$ In general, intercalative interaction separates the local base pairs to accommodate the ligand, thus resulting in DNA lengthening and increased viscosity; groove binding mode does not lead to remarkable changes in DNA viscosity. ${ }^{39}$ Fig. $8 \mathrm{~B}$ showed the slight increase in DNA viscosity changes with the addition of TBZ. The results indicated that TBZ bound to hsDNA though groove rather than through intercalation mode. Therefore, computer simulations and experiments demonstrated that TBZ bound to hsDNA with groove binding mode, which had no effect on the structure of hsDNA. The results were consistent with the study of Jalali et al. ${ }^{26}$

\subsection{Structural changes analysis of DNA}

The infrared spectrum (FT-IR) is usually used to monitor the structure changes of biomacromolecules; the spectra of DNA are mainly confined from $1800 \mathrm{~cm}^{-1}$ to $700 \mathrm{~cm}^{-1}$ region to display protein secondary structure..$^{\mathbf{4 0 , 4 1}}$ The infrared spectral features of the free hsDNA and its complex form interacted with TBZ were shown in Fig. 9A. The band at $1712 \mathrm{~cm}^{-1}$ was related to guanine, which showed no change in wavenumber and intensity. The infrared band observed at $1639 \mathrm{~cm}^{-1}$ (thymine) showed a slight downshift. The infrared bands that appeared at 1541 (guanine and cytosine) and $1498 \mathrm{~cm}^{-1}$ (cytosine) all decreased in terms of intensity at all drug/DNA molar ratios. The deoxyribose sugar vibrations caused by $\mathrm{C}-\mathrm{C}$ stretching were denoted by the infrared bands at $945 \mathrm{~cm}^{-1}$ in the spectrum of free hsDNA. ${ }^{42}$ The new negative band at $1017 \mathrm{~cm}^{-1}$ confirmed that TBZ reacted with hsDNA.
The difference spectra of [(DNA + TBZ) - DNA] (Fig. 9B) showed the remarkable negative band at $1639 \mathrm{~cm}^{-1}$ of thymine with decreasing intensity. The infrared band at $1102 \mathrm{~cm}^{-1}$ was primarily due to the phosphate symmetric vibrations; this band was assigned to the vibrations of the phosphodiester bonds $\left(857 \mathrm{~cm}^{-1}\right) .{ }^{43}$ The new band at $1410 \mathrm{~cm}^{-1}$ appeared when TBZ was added, which indicated that TBZ bound to hsDNA.

The structure of B-form is the closest with DNA in cell. FT-IR can be used to distinguish the DNA structure between B-form and A-form. ${ }^{44}$ The bands at 1461 and $1220 \mathrm{~cm}^{-1}$ were stable, revealing that the DNA conformation tend to be in B-form. In Fig. 9A, the positive band at $893 \mathrm{~cm}^{-1}$ appeared, indicating the partial perturbation of DNA conformation. Moreover, the appearance and disappearance of the band at $861 \mathrm{~cm}^{-1}$ with the addition of TBZ confirmed that the A-form DNA was unstable. Thus, the interaction of the food additive TBZ with hsDNA did not result in transitions in the DNA double helix conformation. hsDNA was in the B-form with the perturbation of B-form transformed into A-form, which meant that the DNA helix became short and compact.

\section{Conclusions}

In this study, the binding mechanism and conformation stability of TBZ to hsDNA were characterized by computer simulations and spectroscopic methods. Computer simulations showed that TBZ could combine with the groove region of DNA through hydrogen bonds and hydrophobic interactions. The thiazole and imidazole rings of TBZ, particularly nitrogen atoms, were the main groups that maintain the stability of the binding with DNA. According to RMSD results, MD simulations were divided into three stages. This finding indicated that TBZ moved from the middle to the terminal region of DNA to form a stable conformation. The mechanism of TBZ binding to DNA was static quenching with weak binding. The binding of TBZ induced the slight conformation changes in DNA. Through comparison of the present results with previous studies, the selection of DNA was important for the study of ligand-receptor 
interaction because of different binding mechanisms. This research provided an in-depth study of the binding mechanism, facilitated the application and development of computer simulations on the interactions of small molecules and biomacromolecules, and provided information on the selection of DNA model from different sources in interaction studies.

\section{Conflicts of interest}

There are no conflicts to declare.

\section{Acknowledgements}

This work was supported by Sichuan Science and Technology Program (Grant No. 2018JY0188), the National Natural Science Foundation of China (NNSFC Grant 21704069), and the Fundamental Research Funds for the Central Universities (Grant No. 2018SCU12043) the Postdoctoral Foundation of Sichuan University.

\section{References}

1 M. Blazková, P. Rauch and L. Fukal, Biosens. Bioelectron., 2010, 25, 2122-2128.

2 N. B. Andrioli and M. D. Mudry, Discrete Appl. Math., 2011, 22, 17-23.

3 N. B. Andrioli, S. Soloneski, M. L. Larramendy and M. D. Mudry, Mutat. Res., Genet. Toxicol. Environ. Mutagen., 2014, 772, 1-5.

4 Y. Feng, M. Lv, Y. Lu, K. Liu, L. Liu, Z. He, K. Wu, X. Wang, B. Zhang and X. Wu, Food Chem., 2017, 243, 118-124.

5 V. Ferraro, A. R. Madureira, B. Sarmento, A. Gomes and M. E. Pintado, Food Res. Int., 2015, 77, 450-459.

6 X. Wang, Y. Liu, L. L. He, B. Liu, S. Y. Zhang, X. Ye, J. J. Jing, J. F. Zhang, M. Gao and X. Wang, Food Chem. Toxicol., 2015, 78, 42-51.

7 A. Opar, Nat. Rev. Drug Discovery, 2009, 8, 437-438.

8 Q. Zhang, Y. Huang, L. Guo, C. Chen, D. Guo, Y. Chen and Y. Fu, New J. Chem., 2014, 38, 4600-4606.

9 N. Shahabadi and M. Maghsudi, Mol. BioSyst., 2013, 10, 338347.

10 A. Boroujeni, Z. Khorasani-Motlagh and M. Noroozifar, J. Biomol. Struct. Dyn., 2016, 34, 414-426.

11 Z. Aramesh Boroujeni, M. Khorasani-Motlagh and M. Noroozifar, J. Biomol. Struct. Dyn., 2016, 34, 414-426.

12 X. Zhou, C. Zhang, G. Zhang and Y. Liao, RSC Adv., 2016, 6, 5408-5418.

13 S. Yilmaz, F. Unal, D. Yüzbaşığlu and M. Celik, Toxicol. Ind. Health, 2014, 30, 926-937.

14 S. Sharma, M. Yadav, S. P. Gupta, K. Pandav and S. Kumar, Chem.-Biol. Interact., 2016, 260, 256-262.

15 C. G. Reinhardt and T. R. Krugh, Biochemistry, 1978, 17, 4845-4854.

16 X. Li and S. Wang, New J. Chem., 2014, 39, 386-395.

17 C. Ràfols, S. Zarza and E. Bosch, Talanta, 2014, 130, 241-250.

18 M. Šimšíková, Arch. Biochem. Biophys., 2016, 593, 69-79.
19 K. J. Czogalla, A. Biswas, K. Höning, V. Hornung, K. Liphardt, M. Watzka and J. Oldenburg, Nat. Struct. Mol. Biol., 2016, 24, 77-85.

20 A. Ahmedbelkacem, L. Colliandre, N. Ahnou, Q. Nevers, M. Gelin, Y. Bessin, R. Brillet, O. Cala, D. Douguet and W. Bourguet, Nat. Commun., 2016, 7, 12777.

21 M. P. Williamson, Prog. Nucl. Magn. Reson. Spectrosc., 2013, 73, 1-16.

22 A. Gossert and W. Jahnke, Prog. Nucl. Magn. Reson. Spectrosc., 2016, 97, 82-125.

23 R. Amiri, A. K. Bordbar, M. F. Garcã-A-Mayoral, A. R. Khosropour, I. Mohammadpoor-Baltork, M. MenãNdez and D. V. Laurents, J. Colloid Interface Sci., 2012, 369, 245-255.

24 D. Wu, Y. Zhai, J. Yan, K. Xu, Q. Wang, Y. Li and H. Li, RSC Adv., 2015, 5, 11036-11042.

25 Y. Wang, T. Zhang, J. Xu and W. Du, Int. J. Biol. Macromol., 2011, 48, 81-86.

26 F. Jalali and P. S. Dorraji, Arabian J. Chem., 2013, 77, S3947S3954.

27 M. Xu, Z. R. Ma, L. Huang, F. J. Chen and Z. Z. Zeng, Spectrochim. Acta, Part A, 2011, 78, 503-511.

28 S. Fujiwara and T. Amisaki, Proteins: Struct., Funct., Bioinf., 2006, 64, 730-739.

29 Y. Cao and X. W. He, Spectrochim. Acta, Part A, 1998, 54, 883892.

30 Q. Sun, H. Yang, P. Tang, J. Liu, W. Wang and H. Li, Food Chem., 2017, 243, 74-81.

31 A. Sharma, S. Anandakumar and M. Ilanchelian, RSC Adv., 2014, 4, 36267-36281.

32 L. Fotouhi and M. Zabeti, Monatsh. Chem., 2016, 147, 837-844.

33 B. Barare, M. Yildiz, H. Ünver and K. Aslan, Tetrahedron Lett., 2016, 57, 537-542.

34 Q. Wang, Q. Sun, P. Tang, B. Tang, J. He, X. Ma and H. Li, RSC Adv., 2015, 5, 81696-81706.

35 M. M. V. Ramana, R. Betkar, A. Nimkar, P. Ranade, B. Mundhe and S. Pardeshi, Spectrochim. Acta, Part A, 2016, 152, 165-171.

36 H. Yang, P. Tang, B. Tang, Y. Huang, X. Xiong and H. Li, RSC Adv. , 2017, 7, 10242-10251.

37 D. Ajloo, M. E. Moghadam, K. Ghadimi, M. Ghadamgahi, A. A. Saboury, A. Divsalar, M. Sheikhmohammadi and K. Yousefi, Inorg. Chim. Acta, 2015, 430, 144-160.

38 N. Shahabadi and S. Amiri, Spectrochim. Acta, Part A, 2015, 138, 840-845.

39 S. Zhang, X. Sun, R. Kong and M. Xu, Spectrochim. Acta, Part A, 2015, 136, 1666-1670.

40 A. A. Ouameur and H. A. Tajmir-Riahi, J. Biol. Chem., 2004, 279, 42041-42054.

41 F. A. Joozdani, F. Yari, P. A. Joozdani and S. Nafisi, PLoS One, 2015, 10, e0127541.

42 D. K. Jangir, G. Tyagi, R. Mehrotra and S. Kundu, J. Mol. Struct., 2010, 969, 126-129.

43 G. Tyagi, S. Charak and R. Mehrotra, J. Photochem. Photobiol., $B, 2012,108,48-52$.

44 L. Han, Y. Zhou, X. Huang, M. Xiao, L. Zhou, J. Zhou, A. Wang and J. Shen, Spectrochim. Acta, Part A, 2014, 123, 497-502. 